\title{
MODELO PARA CLASSIFICAÇÃO DE CARACTERÍSTICAS DE CUSTOMIZAÇÃO EM MASSA PARA SERVIÇOS
}

\section{MODEL TO CLASSIFY SERVICES MASS CUSTOMIZATION CHARACTERISTICS}

\author{
Gabriel Vidor* Email: gvidor@producao.ufrgs.br \\ Janine Fleith de Medeiros ${ }^{\star *}$ Email: janine@upf.br \\ José Luis Duarte Ribeiro* Email: ribeiro@producao.ufrgs.br \\ *Universidade Federal do Rio Grande do Sul, Porto Alegre, RS \\ ${ }^{* *}$ Universidade de Passo Fundo, Passo Fundo, RS
}

\begin{abstract}
Resumo: Este trabalho teve por objetivo verificar quais e como são as relações entre as características de customização em massa e as estratégias de serviços. Para tanto, foi organizado um estudo que mapeia as características de customização em massa e as classifica para a área de serviços. A classificação é apresentada através de um framework que utilizou a variável nível de customização como critério de classificação. Pode-se observar que nem todas as características da customização em massa usadas para manufatura podem ser aplicadas na área de serviços. Além disso, as características de manufatura classificadas em qualquer nível de customização, não podem ser replicadas em sua totalidade quando aplicada aos serviços. No fim do estudo, verificam-se também pontos fortes e fracos do framework gerado em função da variável nível de customização. Dois casos de análise ilustram a aderência do framework as práticas organizacionais.
\end{abstract}

Palavras-chave: Customização em massa. Serviços. Classificação.

Abstract: The objective of this paper is to verify the relationship between mass customization characteristics and services strategies. Therefore, a study mapping mass customization characteristics and categorizing them into service area was carried out. The categorization was established through a framework that uses the level of customization as classification criteria. It was observed that not all mass customization characteristics used for manufacturing can be applied to the service sector. Moreover, manufacturing characteristics classified at any level of customization may not be fully replicated when applied to services. At the end of the study, strengths and weaknesses of the framework are evaluated according to levels of customization. Two cases were analyzed to illustrate the adherence of the framework to organizational practices.

Keywords: Mass customization. Service. Classification.

\section{INTRODUÇÃO}

Em função de sua contribuição em termos operacionais e de gestão para o cotidiano das organizações, a customização em massa (CM) tem despertado contínuo

Revista Produção Online, Florianópolis, SC, v.13, n. 3, p. 974-1001, jul./set. 2013. 
interesse da academia e de empresas (TRENTIN et al., 2012). Contudo, apesar do tema apresentar soluções potenciais para área de manufatura, com estudos consolidados nas áreas de cadeia de suprimentos (ABDELKAFI et al., 2010), análise de mercado e planejamento da estrutura de produtos (SPRING e ARAUJO, 2009) e planejamento e controle da produção (ZHANG e CHEN, 2006; TSENG e RADKE, 2010; LEE e DAÍ, 2010), ainda existem questionamentos sobre a real aplicabilidade da customização em massa na área de serviços (McCARTHY et al., 2010).

Em parte essas questões originam-se da subjetividade do termo customização em massa e, por outro lado, das peculiaridades da gestão em serviços considerando suas características distintivas. Sabe-se que o conceito de CM tem sido aplicado em diversos contextos, tanto na prestação de serviços quanto na manufatura (ANZANELLO e FOGLIATOO, 2011). Todavia, a aplicabilidade desse conceito faz com que as apropriações em relação ao termo customização em massa tornem-se diversas e, mesmo que complementares, com algumas diferenças em relação ao conceito original estabelecido por Davis em 1987 (ARAUJO e GOMES, 2010). Segundo o referido autor, CM refere-se a uma resposta rápida da indústria automotiva para as escolhas do cliente. De fato, conceitualmente a CM pode ser entendida como uma estratégia de negócios que diferencia as organizações em ambientes de alta competitividade e torna mais qualificada a segmentação de mercado (HELO et al., 2010).

Apesar da evolução observada no conceito original, as soluções apresentadas na literatura para $\mathrm{CM}$ na área de serviços ainda são incipientes (PETERS e SAIDIN, 2000). Isso porque o setor de serviços apresenta altos graus de intangibilidade, perecibilidade, inseparabilidade e variabilidade (REUVER e BOUWMAN, 2012). Assim, os serviços customizados caracterizam-se essencialmente por: (i) heterogeneidade de demandas de mercado, (ii) velocidade e variedade exigida pelos clientes nesse tipo de segmento e (iii) competição com empresas do mesmo segmento (CAO et al., 2010). Combinado ao nível de customização (PAN e HOLLAND, 2006; SILVEIRA et al., 2000), esses aspectos acabam dificultando a vinculação entre serviços e customização em massa.

Revista Produção Online, Florianópolis, SC, v.13, n. 3, p. 974-1001, jul./set. 2013. 
De fato, a área de serviços customizados apropria-se das características de manufatura customizada, não havendo uma classificação das características de customização sob a perspectiva de serviços. Salienta-se, contudo, que uma abordagem para obter características de serviços customizados seria utilizar as características de manufatura e, através da variável de nível de customização, verificar quais podem ser utilizadas para caracterizar os "serviços customizados em massa" (McCARTHY et al, 2010). Nesse sentido, o objetivo desse trabalho é verificar quais e como são as relações entre as características de customização em massa e as estratégias de serviço. Em suma, é proposta uma classificação de características de customização para a área de serviços, tendo-se como critério principal o nível de customização.

A contribuição deste artigo para área de conhecimento em Engenharia de Produção está no fato de fornecer elementos que permitem alinhar estratégias de produção, no caso a customização em massa, com a gestão de serviços. Essa integração tem sido estudo frequente em casos da engenharia de produção (PRIETO et al., 2007).

Para tanto, o trabalho está organizado em cinco seções. Além dessa introdução, a segunda seção aborda uma revisão dos níveis de CM existentes e as características da estratégia de CM. Nessa seção é realizada uma descrição das características distintivas dos serviços e suas implicações gerenciais. Na terceira seção tem-se a apresentação do método utilizado e das etapas desenvolvidas no trabalho. $\mathrm{Na}$ quarta seção mostra-se o framework elaborado e é realizada uma discussão sobre a classificação realizada. Dois casos de estudo são usados para ilustrar o estudo. Finalmente, a última seção apresenta as conclusões do trabalho, bem como a sugestão de estudos futuros na área.

\section{REFERENCIAL TEÓRICO}

\subsection{Níveis de customização}


Os níveis de customização podem ser classificados quanto ao produto e ao processo (SILVEIRA et al., 2001) e quanto ao envolvimento do cliente (PAN e HOLLAND, 2006).

Pan e Holland (2006) definem seis níveis de customização em função da relação do cliente com o processo. Os níveis, em grau crescente de customização, são: captação do cliente, alteração, superficialidade, transparência, adaptação e colaboração. O primeiro nível, de captação do cliente, consiste em entender o mercado e repensar o sistema de manufatura, permitindo que os clientes encontrem o que querem através de uma ampla oferta de produtos. O segundo nível, de alteração, pressupõe que os funcionários da empresa aprendam sobre customização e a pratiquem como diferencial para os clientes. Duray (2002) ressalta ser especialmente crítica a transição para esse nível, haja vista a complexidade da mudança de cultura. $O$ terceiro nível, de superficialidade, é o nível em que a cadeia de suprimento é envolvida, com a mudança nos sistemas de distribuição e entrega de itens para o cliente final.

No nível posterior, de transparência, proporcionam-se aos clientes produtos únicos sem necessariamente fazer customização explícita. Como definem $\mathrm{Ni}$ et al. (2008) este é o nível de modularização, com a customização das partes. No quinto nível, de adaptação, clientes começam a se envolver no desenvolvimento do projeto adicionando informações à manufatura e pós-vendas (customização do serviço associado ao produto). Por fim, no último nível, de colaboração, os clientes são desenvolvedores do projeto do produto ou serviço, inclusive com interações computacionais em um espaço de soluções e, através dessas interações, caracterizando o produto ou serviço. Fiori (2000) define esse nível como o mais alto de $\mathrm{CM}$, pois permite que o cliente seja parte integrante do desenvolvimento que irá usufruir.

Fogliatto et al. (2012) identificaram oito níveis de customização para produtos e processos. Esses níveis apresentam a seguinte ordem decrescente: projeto, fabricação, montagem, adição de trabalho customizado, adição de serviços, embalagem e distribuição, uso, padronização.

Revista Produção Online, Florianópolis, SC, v.13, n. 3, p. 974-1001, jul./set. 2013. 
O nível de projeto é o nível de desenvolvimento colaborativo de projeto, manufatura e entrega de produtos de acordo com as necessidades preferenciais do cliente. O nível de fabricação refere-se à fabricação de produtos adaptados ao cliente a partir de projetos iniciais. O nível de montagem oferta produtos através de arranjos modulares de acordo com diferentes demandas do cliente.

Os níveis de adição de trabalho e serviço são obtidos com a complementação de trabalho em produtos padronizados, que ocorre anteriormente a entrega para cliente. O nível de embalagem e distribuição é obtido com a diferenciação de produtos similares através da adequação de embalagens e caminhos de entrega pelo tipo de mercado consumidor. O nível de uso ocorre quando o produto é aplicado diferentemente pelos clientes finais, ou seja, quando há uma adaptação do produto. Finalmente a padronização é a prática conhecida e usada em muitas organizações.

A análise desses dois grupos mostra a existência de uma convergência independentemente da visão escolhida. Os níveis de colaboração e adaptação são similares aos níveis de projeto e fabricação; logo, neste caso, pode-se estabelecer o estágio de projeto e processo como o estágio alto de customização. Em um estágio médio-alto poderiam ser agrupados os níveis de montagem e transparência, que poderia ser chamado de montagem, dado que este termo é comumente conhecido, quando comparado a transparência. Em um estágio médio-baixo poderiam ser incluídos os níveis de adição de trabalho e serviço, embalagem e distribuição e superficialidade, passando esse estágio a ser denominado postponement. Por fim, no estágio baixo, poderia ser enquadrados os níveis de adaptação e cosmético, onde os níveis de alteração e uso estariam contemplados. Os níveis de padronização e captação não fazem parte dos níveis definidos, dado que são elementos associados a PM e não a CM.

Assim, de maneira resumida, é possível considerar que os níveis de customização existentes poderiam ser classificados em alto - projeto e processo, médio-alto - montagem, médio-baixo - postponement, e baixo - adaptação e cosmético.

Revista Produção Online, Florianópolis, SC, v.13, n. 3, p. 974-1001, jul./set. 2013. 


\subsection{Características de customização em massa}

Para obter as características de customização citadas na sequência desse trabalho, foi realizada uma revisão da literatura, através de uma varredura horizontal, onde foram identificados os diversos aspectos da CM. A revisão do tema de CM foi realizada nas bases de dados Emerald, Sage, ScienceDirect, Springer-Verlag \& Kluwer, Elsevier. A pesquisa foi executada a partir da palavra chave "mass customization", e o período para a busca foi restringido de 1990 até 2010. A restrição do período deve-se ao fato do termo mass customization ter sido utilizada pela primeira vez no ano de 1987 por Davis, não havendo publicações correlatas ao assunto em períodos anteriores. A partir da base de artigos obtidos foi realizada uma meta-análise para verificar quais poderiam ser aproveitados para confeccionar esse estudo.

Dessa forma, foram identificadas 13 características de customização, em um total de 83 artigos. Para desdobrar claramente as características que estão associadas ao conceito de $\mathrm{CM}$, foi organizado um quadro, apresentado na Figura 1, que mostra essas diferentes características.

Revista Produção Online, Florianópolis, SC, v.13, n. 3, p. 974-1001, jul./set. 2013. 
Figura 1 - Características de customização em massa

\begin{tabular}{|c|c|c|}
\hline \multicolumn{2}{|r|}{ CARACTERÍSTICAS } & REFERÊNCIAS \\
\hline C1 & $\begin{array}{llr}\text { A estrutura/arquitetura } & \text { de serviço } \\
\text { organizada } & \text { em remilias, } \\
\text { plataformas, } & \text { módulos } & \text { e } \\
\text { componentes. } & & \end{array}$ & $\begin{array}{l}\text { Tseng e Jiao (1996), Fischer et al. (1999), Jiao e Tseng (1999), Muffato (1999), Dahmus et } \\
\text { al. (2001), Simpson et al. (2001), Gershenson et al. (2003), Simpson (2004), Zha et al. (2004), } \\
\text { Corbett e Rosen (2004), Jiao e Tseng (2004), Jose e Tollenaere (2005), Huang et al. (2005), } \\
\text { Zhang et al. (2005), Marion et al. (2006), Simpson et al. (2006), Thevenot e Simpson (2006), } \\
\text { Dai e Scott (2007), Huang et al. (2007), Jiao et al. (2007), Alizzon et al. (2007), Mun et al. } \\
\text { (2007), Willians et al. (2007), Fixson (2007), Li et al. (2008), Ni et al. (2008), Lindquist et al. } \\
\text { (2008), Kumar (2008), Kumar et al. (2008), Salvador et al. (2009). }\end{array}$ \\
\hline $\mathbf{C 2}$ & $\begin{array}{l}\text { Projetos adaptáveis obtidos por } \\
\text { processos customizados. }\end{array}$ & $\begin{array}{l}\text { Tseng e Jiao (1996), Jiao e Tseng (1999), Duray et al. (2000), Dahmus et al. (2001), Duray } \\
\text { (2002), Karlsson (2002), Piller (2004), Jiao e Tseng (2004), Corbett e Rosen (2004), Zha et } \\
\text { al. (2004), Gershenson (2004), Zhang et al. (2005), Jose e Tollenaere (2005), Pan e Holland } \\
\text { (2006), Dai e Scott (2007), Alizon et al. (2007), Ismail et al. (2007), Apeagyei e Otieno } \\
\text { (2007), Willians et al. (2007), Lindquist et al. (2008), Bare e Cox (2008), Li et al. (2008). }\end{array}$ \\
\hline C3 & $\begin{array}{l}\text { Integração do cliente no processo } \\
\text { de serviço, permitindo total } \\
\text { atendimento de suas necessidades } \\
\text { e expectativas. }\end{array}$ & $\begin{array}{l}\text { Duray et al. (2000), Da Silveira et al. (2001), Connell et al. (2002), Bardacki e Whitelock } \\
\text { (2003), Siddique e Boddu (2004), Zha et al. (2004), Bardacki e Whitelock (2004), Piller et al. } \\
\text { (2004), Piller (2004), Bardacki e Whitelock (2005), Sigala (2006), Willians et al. (2007), Wang } \\
\text { e Lin (2008), Haug et al. (2009), Cho e Fiorito (2009), Spring e Araujo (2009). }\end{array}$ \\
\hline $\mathrm{C4}$ & $\begin{array}{l}\text { Planejamento de marketing e } \\
\text { marketing do serviço. }\end{array}$ & $\begin{array}{l}\text { Duray et al. (2000), Connel et al. (2002), Bardacki e Whitelock (2003), Mun et al. (2007), } \\
\text { Endo e Kincade (2008). }\end{array}$ \\
\hline C5 & $\begin{array}{|lrr|}\text { Sistemas de informação } & \text { que } \\
\text { funcionem como canais } & \text { de } \\
\text { comunicação. } & & \\
\end{array}$ & $\begin{array}{l}\text { Jiao e Tseng (2004), Piller (2004), Siddique e Boddu (2004), Jiao et al. (2007), Alizon et al. } \\
\text { (2007), Ma et al. (2008), Ni et al. (2008), Lindquist et al. (2008), Fogliatto e Da Silveira } \\
\text { (2008), Feng et al. (2008). }\end{array}$ \\
\hline C6 & $\begin{array}{l}\begin{array}{l}\text { Customização } \\
\text { suprimentos. }\end{array} \\
\text { da }\end{array}$ & $\begin{array}{l}\text { al. (2002), Yang (2004), Pan e Holland (2006), Huang et al. (2005), } \\
\text { quist (2008), Jitpaiboon et al. (2009). }\end{array}$ \\
\hline C7 & $\begin{array}{l}\text { Gestão de dados, configurações, } \\
\text { etapas da prestação de serviço. }\end{array}$ & t al. (2008), Liou et al. (2010). \\
\hline $\mathrm{C} 8$ & $\begin{array}{|llll|}\begin{array}{l}\text { Determinação de custos por } \\
\text { atividades. }\end{array} & \\
\end{array}$ & 2007). \\
\hline C9 & $\begin{array}{l}\text { Integração entre planejamento dos } \\
\text { serviços e as metas da } \\
\text { organização. }\end{array}$ & $\begin{array}{l}\text { Duray et al. (2000), Duray (2002), Salvador et al. (2002), Brown e Bessant (2003), Jiao e } \\
\text { Tseng (2004), Du et al. (2005), Alizon et al. (2007), Lindquist et al. (2008), Jitpaiboon et al. } \\
(2009) \text {, Starr (2010). }\end{array}$ \\
\hline C10 & $\begin{array}{ll}\begin{array}{l}\text { Agilidade e velocidade da } \\
\text { prestação de serviço. }\end{array} & \\
\end{array}$ & Silveira et al. (2001), Brown e Bessant (2003), Ismail et al. (2007). \\
\hline C11 & $\begin{array}{l}\text { Ferramentas } \\
\text { concorrente, sistemas hibridos, } \\
\text { etc.) e estratégias de produção } \\
\text { (produção enxuta, produção em } \\
\text { massa, etc.). }\end{array}$ & $\begin{array}{l}\text { Kotha (1995), Muffatto (1999), Fisher et al. (1999), Hoek (2001), Salvador et al. (2002), } \\
\text { Karlsson (2002), Piller et al. (2004), Simpson (2004), Bardacki e Whitelock (2005), Jose e } \\
\text { Tollenaere (2005), Zhang et al. (2005), Zhang e Chen (2006), Simpson et al. (2006), Mun et } \\
\text { al. (2007), Kincade et al. (2007), Apeagyei e Otieno (2007), Bayraktar (2007), Alizon et al. } \\
\text { (2007), Jiao et al. (2007), Ni et al. (2008). }\end{array}$ \\
\hline $\mathrm{C} 12$ & $\begin{array}{|lr|}\text { Criação de conhecimento através } \\
\text { de } & \text { retroalimentação } \quad \text { de } \\
\text { informações. }\end{array}$ & Kotha (1995), Zha et al. (2004). \\
\hline C13 & $\begin{array}{|lrr|}\begin{array}{l}\text { Técnicas } \\
\text { padronização, }\end{array} & \text { prototipagem } & \text { e } \\
\text { personalização. } & & \\
\end{array}$ & $\begin{array}{l}\text { Tseng e Jiao (1996), Bardacki e Whitelock (2004), Piller (2004), Bare e Cox (2008), Piller } \\
\text { (2008), Kumar (2008). }\end{array}$ \\
\hline
\end{tabular}

Fonte: Elaborado pelos autores

Todas as características prevalentes estão representadas na Figura 2. As características mais referenciadas nos estudos estão associadas à definição de estrutura de produto (C1), à utilização de projetos e processos adaptáveis (C2), ao tipo de estratégia de manufatura (C11) e ao atendimento das necessidades de clientes (C3), correspondendo, respectivamente, a 43,5\%, 32\%, 29\% e 23\%. Considerando o 
exposto, a Figura 2 apresenta o total (em percentual) de artigos que abordam as características em relação à quantidade total de artigos usados para a análise.

Figura 2 - Frequência de características citadas em estudos de CM



Fonte: elaborado pelos autores

As discussões realizadas até o momento proporcionam a definição de um conceito de $\mathrm{CM}$, a ser utilizado neste artigo. Dessa forma, entende-se customização em massa como a capacidade de ofertar produtos e serviços com grau de variedade que atenda às necessidades do cliente e apresentem custos similares aos de produção em massa. A oferta de grande quantidade de produtos e serviços garante que a empresa seja capaz de atender aos requisitos de customização, o primeiro binômio do elemento customização/massa. Os custos similares ao da produção em massa garantem que a empresa atenda ao segundo elemento: o da produção em massa, ofertando altos volumes dentro de um custo que racionalize a operação de manufatura e a prestação do serviço. Essa definição serve para nortear as discussões do trabalho, não sendo um conceito definitivo para o termo. 


\subsection{A gestão de serviços}

Serviços diferenciam-se dos bens em decorrência de quatro características específicas: (i) intangibilidade, (ii) perecibilidade, (iii) variabilidade e (iv) inseparabilidade (HUEMER, 2012). Isto quer dizer que a natureza dos processos planejados para a gestão de serviços apresenta particularidades quando comparado aos processos de administração e entrega ao mercado de bens físicos (ZEITHAML, 1981).

A intangibilidade (i) corresponde ao fato de que em serviços o processo é o produto (KOTLER, KELLER, 2006). Por isso, os consumidores de serviços buscam por evidências da qualidade do serviço, tais como instalações, pessoas, equipamentos, material de divulgação e preço (BOOMS, BITNER, 1981; LEVITT, 1990). Já perecibilidade (ii) diz respeito à incapacidade de estoque dos serviços. Assim, os gestores de organizações prestadoras de serviços devem promover ações que busquem proporcionar um equilíbrio entre oferta e demanda, tais como preços diferenciados, ações promocionais, serviços complementares e mecanismos de reserva (FITZSIMMONS, FITZSIMMONS, 2010).

Com relação à variabilidade (iii), esta se refere à extrema dependência que os serviços possuem das pessoas. Dessa forma, as organizações que atuam no setor devem investir em processos de contratação e treinamento compatíveis com o posicionamento da empresa, devem padronizar o processo de execução do serviço, e devem monitorar a satisfação dos clientes (ZAHAY, GRIFFIN, 2002). Por fim, a inseparabilidade trata da simultaneidade entre prestador e consumidor, quase sempre necessária (LOVELOCK, WRIGHT, 2011).

Obviamente, o impacto das referidas características possui relação direta com o grau de interação e personalização dos serviços (FITZSIMMONS, FITZSMMONS, 2010). Isto quer dizer que os gestores envolvidos na entrega de serviços aos distintos mercados consumidores devem focar suas decisões na minimização dos efeitos das características específicas do processo de serviços que controlam, ou seja, 
considerando a intensidade de trabalho e a interação necessária, como se observa na Figura 3.

Figura 3 - Modelo de gestão de serviços

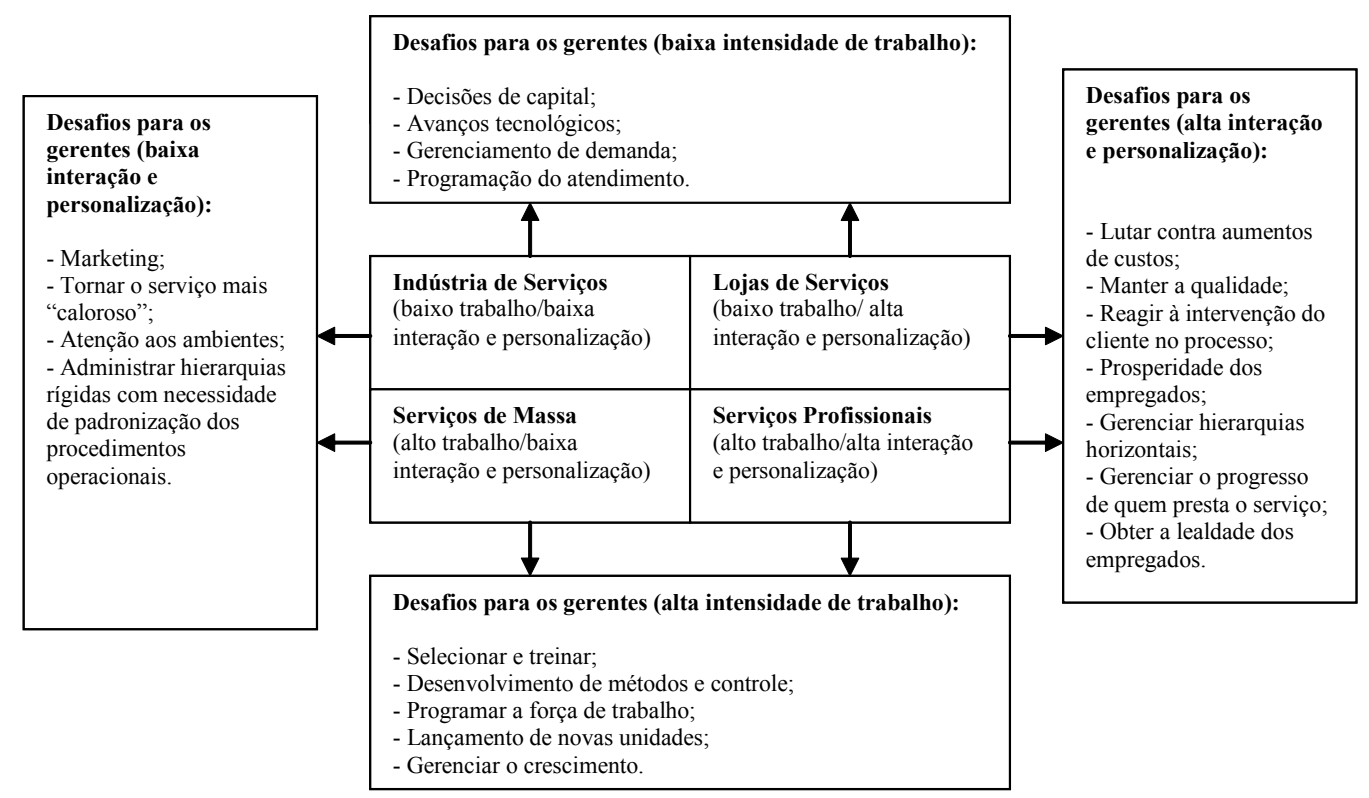

Fonte: adaptado de Schmenner (apud FITZSMMONS, FITZSMMONS, 2010)

\section{METODOLOGIA}

A presente pesquisa pode ser classificada como uma pesquisa qualitativa, na qual se utilizou meta-análise, estruturada através da revisão de casos da literatura do tema "níveis e características da customização em massa". A pesquisa é de natureza triangular, o que permitiu efetuar comparações entre práticas usadas em empresas e a literatura, para sustentar as conclusões obtidas no estudo. O estudo dos casos da literatura teve por objetivo identificar as características de CM e entender como a sua aplicação pode ser generalizada para o ambiente de serviços, o que demandou uma análise detalhada das características da manufatura. A comparação com as práticas empresariais teve por objetivo validar conceitualmente o framework gerado, permitindo explanações sobre seus pontos fortes e fracos. O framework é assim denominado,

Revista Produção Online, Florianópolis, SC, v.13, n. 3, p. 974-1001, jul./set. 2013. 
porque organiza a classificação de características de customização em função da variável nível de customização.

O estudo foi organizado através de quatro etapas: (i) mapeamento da literatura, para identificar níveis e características da CM; (ii) classificação das características de CM associadas a serviço; (iii) elaboração do framework (modelo de classificação); e (iv) discussão sobre pontos fortes e fracos do modelo de classificação baseado em dois casos de análise.

$\mathrm{Na}$ etapa mapeamento da literatura (i), foi possível identificar as 13 características que, posteriormente, através da variável de nível de customização, foram usadas para montar as estratégias de serviços. Essas características foram obtidas da análise de 83 artigos. Na sequência (ii), essas informações foram validadas com a comparação de características observadas em sistemas reais de prestação de serviços customizados, buscando qualificar e validar as informações que comporiam o framework teórico. Essa etapa foi realizada comparando as referências teóricas com fontes de evidências coletadas de visitas técnicas em sete diferentes organizações de serviços.

Para a elaboração do framework (iii), foram agrupadas em uma tabela todas as características de customização em massa para a área de serviços. A intensidade e a aplicabilidade dessas características foram definidas de acordo com o nível de customização. Isso foi importante, uma vez que permitiu demonstrar como cada característica pode auxiliar no desenvolvimento do serviço customizado em uma organização. Por fim, a discussão sobre os pontos fortes e fracos do modelo de classificação elaborado (iv) foi realizada tendo por referência as características dos serviços, bem como os desafios dos gestores para administrar a matriz de processos de serviços (proposta conceitualmente na Figura 3). Dois casos são usados para ilustrar a efetividade do modelo proposto. 


\section{RESULTADOS}

\subsection{Modelo de classificação}

O modelo foi estruturado de forma a contemplar as características de customização discutidas anteriormente. Buscou-se definir se as características de customização impactam na área de serviços e, em caso positivo, verificar em qual nível de customização elas podem ocorrer. No caso, uma característica pode ser encontrada em apenas um ou em todos os níveis de customização. Isso ocorre porque as características variam em sua intensidade de aplicação. A Figura 4 apresenta a classificação das características, bem como o nível em que cada característica pode ocorrer.

Figura 4 - Classificação das características de customização

\begin{tabular}{|c|c|c|c|c|c|c|}
\hline \multirow{3}{*}{ Caracte rís ticas } & \multirow{3}{*}{ Descrição res umida } & \multirow{3}{*}{ Atendimento } & \multicolumn{4}{|c|}{ Nível de customização } \\
\hline & & & Alto & Médio-alto & Médio-baixo & \\
\hline & & & $\begin{array}{l}\text { Projeto e } \\
\text { processo }\end{array}$ & Montagem & Postponement & $\begin{array}{c}\text { Adaptação e } \\
\text { cosmético }\end{array}$ \\
\hline $\mathrm{C} 1$ & Estrutura do serviço & O & $\mathrm{X}$ & $\mathrm{X}$ & $\mathrm{X}$ & $\mathrm{X}$ \\
\hline $\mathrm{C} 2$ & Projetos adaptáveis & O & $\mathrm{X}$ & $\mathrm{X}$ & & \\
\hline $\mathrm{C} 3$ & Integração do cliente & O & $\mathrm{X}$ & & & \\
\hline $\mathrm{C} 4$ & Marketing do serviço & O & $\mathrm{X}$ & $\mathrm{X}$ & $\mathrm{X}$ & $\mathrm{X}$ \\
\hline $\mathrm{C} 5$ & Canais de comunicação & O & $\mathrm{X}$ & $\mathrm{X}$ & $\mathrm{X}$ & $\mathrm{X}$ \\
\hline C6 & Customização da cadeia & O & & & & \\
\hline $\mathrm{C7}$ & Gestão de dados & O & $\mathrm{X}$ & $\mathrm{X}$ & $\mathrm{X}$ & $\mathrm{X}$ \\
\hline $\mathrm{C} 8$ & Custos por atividades & O & $\mathrm{X}$ & & & \\
\hline C9 & Planejamento e metas da organização & O & & & & \\
\hline $\mathrm{C} 10$ & Agilidade na prestação do serviço & O & $\mathrm{X}$ & $\mathrm{X}$ & $\mathrm{X}$ & $\mathrm{X}$ \\
\hline $\mathrm{C} 11$ & Ferramentas e estratégias de produção & O & $\mathrm{X}$ & $\mathrm{X}$ & $\mathrm{X}$ & $\mathrm{X}$ \\
\hline $\mathrm{C} 12$ & Retroalimentação de informações & 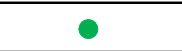 & $\mathrm{X}$ & $\mathrm{X}$ & $\mathrm{X}$ & $\mathrm{X}$ \\
\hline $\mathrm{C} 13$ & Técnicas de repetição & O & $\mathrm{X}$ & & & \\
\hline
\end{tabular}

Fonte: elaborado pelos autores

Assim, a Figura 4 está organizada de forma a mostrar, na coluna da esquerda, as características de customização. Essas características estão identificadas por um

Revista Produção Online, Florianópolis, SC, v.13, n. 3, p. 974-1001, jul./set. 2013. 
número precedido da letra C. Na parte superior da Figura 4, estão dispostos os níveis de customização em que as características podem ser classificadas e, no corpo da tabela, está assinalado se ela se aplicada à área de serviços e também em que nível pode ocorrer. Além disso, há uma coluna chamada atendimento, que mostra se a característica pode ser adaptada para a área de serviços em todos os níveis de customização (verde), em parte dos níveis (amarelo) ou em nenhum nível (vermelho).

Uma primeira análise evidencia que as características de customização da manufatura podem ser utilizadas para o setor de serviços. Isso remete a avaliação de que soluções empregadas para a customização em massa na área de manufatura também podem ser empregadas para a área de serviços dada a paridade de aplicação. Entretanto, cabe ressaltar que, enquanto na área de manufatura as características podem transitar livremente em função do nível de customização, na área de serviço isso não é totalmente possível.

De fato, poder-se-ia atribuir isso as características dos serviços. A intangibilidade, por exemplo, contribuiria para eliminação dos níveis de customização, visto que esse nível passa por vezes pela interpretação do cliente. A variabilidade e


nível de customização.

A característica $\mathrm{C} 1$ é totalmente aplicável à área de serviços, porque um serviço pode ser organizado em diferentes níveis de atendimento. No nível de projeto e processo, podem ser classificados os serviços personalizados; no nível médio-alto, os serviços que combinam opções de módulos de produto; nos serviços médio-baixo, estariam os módulos de serviço com ligeiras modificações de atividades nesses módulos; e no último nível o produto padrão, com atendimento customizado (conforme estabelece a matriz de processos dos serviços).

Por exemplo, na venda de serviços bancários, o nível alto seria para atender clientes que demandam atividades realizadas exclusivamente para eles, sem padronização de atendimento ou rotinas. No nível médio-alto estariam os clientes que podem escolher diversos módulos, combinando atividades desses módulos. No serviço 
médio-baixo o cliente teria que escolher por algum módulo de serviços ofertado pelo banco e combiná-los a poucas atividades de outros módulos. Finalmente, no nível baixo o cliente optaria por uma plataforma de serviços prestados de forma padrão.

A característica C2 aplica-se apenas aos níveis mais altos de customização, dado a onerosidade necessária para sua aplicação em níveis inferiores. De fato, não faz sentido envolver o cliente para customizar um serviço em baixo nível, pois, no caso de customização do serviço, isso será praticamente em sua totalidade, ou então com a combinação de muitos módulos. Por exemplo, em serviços de limpeza o cliente solicita um tipo de procedimento com todas as características que busca, ou no máximo escolhe módulos, como limpar vidros, encerar, varrer (a gestão desta característica na customização auxiliaria na redução do impacto da perecibilidade).

A característica C3 aplica-se apenas para o maior nível de customização, visto que o serviço atende ou não às expectativas do cliente. Não há como atender parcialmente ou em algum nível. Todavia, a característica C4 apresenta-se para todos os níveis de customização. O plano de marketing pode ser realizado em diferentes níveis e pode ser definido por cada cliente, seguindo modelos de referência, com pesquisas de mercado e malas diretas, e até com criação de marcas e condicionamento do planejamento estratégico da organização.

Tal qual a característica C4, a característica C5 também pode ser aplicada nos diversos níveis de customização. O cliente pode transitar da ouvidoria do serviço ao abastecimento do sistema de informação da organização com seu conhecimento. $O$ entendimento pleno do serviço, por vezes, pode estar apenas em domínio do cliente, não sendo possível que o prestador do serviço abstraia o que o cliente busca. Dessa forma, o cliente pode interagir em reuniões com grupos da empresa, ou através de contato via sistema, e auxiliar na customização do serviço desejado. Por exemplo, na compra de aulas de inglês, o cliente pode escolher o produto padrão e, através da ouvidoria, pode informar sobre expectativas do curso ou ajudar na confecção da ementa e ter suas expectativas atendidas anteriormente à prestação do serviço (o que reduziria a intangibilidade).

Revista Produção Online, Florianópolis, SC, v.13, n. 3, p. 974-1001, jul./set. 2013. 
A característica C6 não apresenta a relação correspondente entre manufatura e serviço. Enquanto na área de manufatura a relação de fornecimento pode ser determinada em diversos níveis e intensidade, na área de serviço isso não faz sentido, visto que a customização do serviço não depende da customização do fornecedor de insumos para o serviço. Contudo, a característica C7 é aplicável a qualquer nível de customização, porque independe do serviço ou do produto. O controle de dados ocorre em relação ao processo, portanto pode ser realizado em qualquer nível e também ser usado para qualquer situação em que seja necessário o controle (o que auxilia na minimização do impacto da variabilidade).

A característica C8 foi classificada apenas no nível máximo de customização, pois o controle de custos na $\mathrm{CM}$ é usado através do método $\mathrm{ABC}$. Dessa forma, o detalhamento das atividades já é realizado em seu nível máximo, o que torna improdutivo usar um controle em nível de customização menor. Além disso, para a área de serviços, os controles devem ser realizados por atividades, sendo ineficiente o controle de custos em outros níveis de customização. Por sua vez, a característica C9 também não é aplicável ao setor de serviços. Apesar de existir indicadores de processo utilizados para a área, não é viável a utilização de roteiros para a prestação de todos os tipos de serviço conforme identificado na literatura em decorrência do grau de interação e personalização, bem como do grau de intensidade da mão-de-obra.

A característica C10 é aplicável em todos os níveis de customização de serviços. A agilidade é medida pelo tempo de prestação do serviço, e a CM permite a organização do serviço de forma planejada e focada em pequenas padronizações de atividades ou grupos de atividades (o que auxilia a evitar a perecibilidade e favorece consumidor e prestador). Além disso, a utilização de estratégias de produção, como produção enxuta e produção em massa, às ferramentas da produção, como a engenharia concorrente e teoria das restrições, proporcionadas pela $\mathrm{CM}$, fazem com que a característica C11 seja aplicada também em todos os níveis de customização. Exemplificando: no nível alto de customização, as escolhas do cliente influem na seleção da ferramenta de controle da qualidade final do serviço; no nível médio-alto, 
curvas $A B C$ seriam usadas para determinar quais as tarefas podem ser customizadas $e$ quais devem ser usadas de forma padronizada. Já no nível médio-baixo, a forma de execução dos serviços poderia ser definida a partir de uma survey com o cliente, anteriormente a execução; e, no nível baixo, a escolha de tipos de serviço diretamente determina o grupo de ferramentas utilizadas.

A característica C12 aplica-se a todos os níveis de conhecimento, pois a criação do conhecimento na organização pode estar distribuída em diversos níveis, áreas e acontecer independentemente da complexidade de um processo. Finalmente, a característica $\mathrm{C} 13$ só pode se utilizada no maior nível de customização. O serviço, mesmo que apresente rotinas, nunca poderá ser totalmente padronizado. Dessa forma, mesmo que as técnicas de repetição, por exemplo, sejam priorizadas, as atividades não acontecem exatamente da mesma forma (serviços são variáveis e intangíveis).

Conforme a análise realizada, verifica-se que características de customização em massa para manufatura podem ser adaptadas para a customização em massa de serviços, desde que se respeitem os graus de interação dos processos de serviços e suas características singulares. Para consolidar as contribuições aqui descritas, sugerese como próximo passo testar, através de um estudo de caso múltiplo, a aderência das características propostas no framework. Contudo, destaca-se que as leituras preliminares de estudos de caso existentes na literatura demonstram que a relação entre a área de serviços e as características distribuídas no framework desse trabalho são viáveis, como pode ser exemplificado através da Figura 5.

Revista Produção Online, Florianópolis, SC, v.13, n. 3, p. 974-1001, jul./set. 2013. 
Figura 5 - Exemplos da aplicação das características de CM em serviços

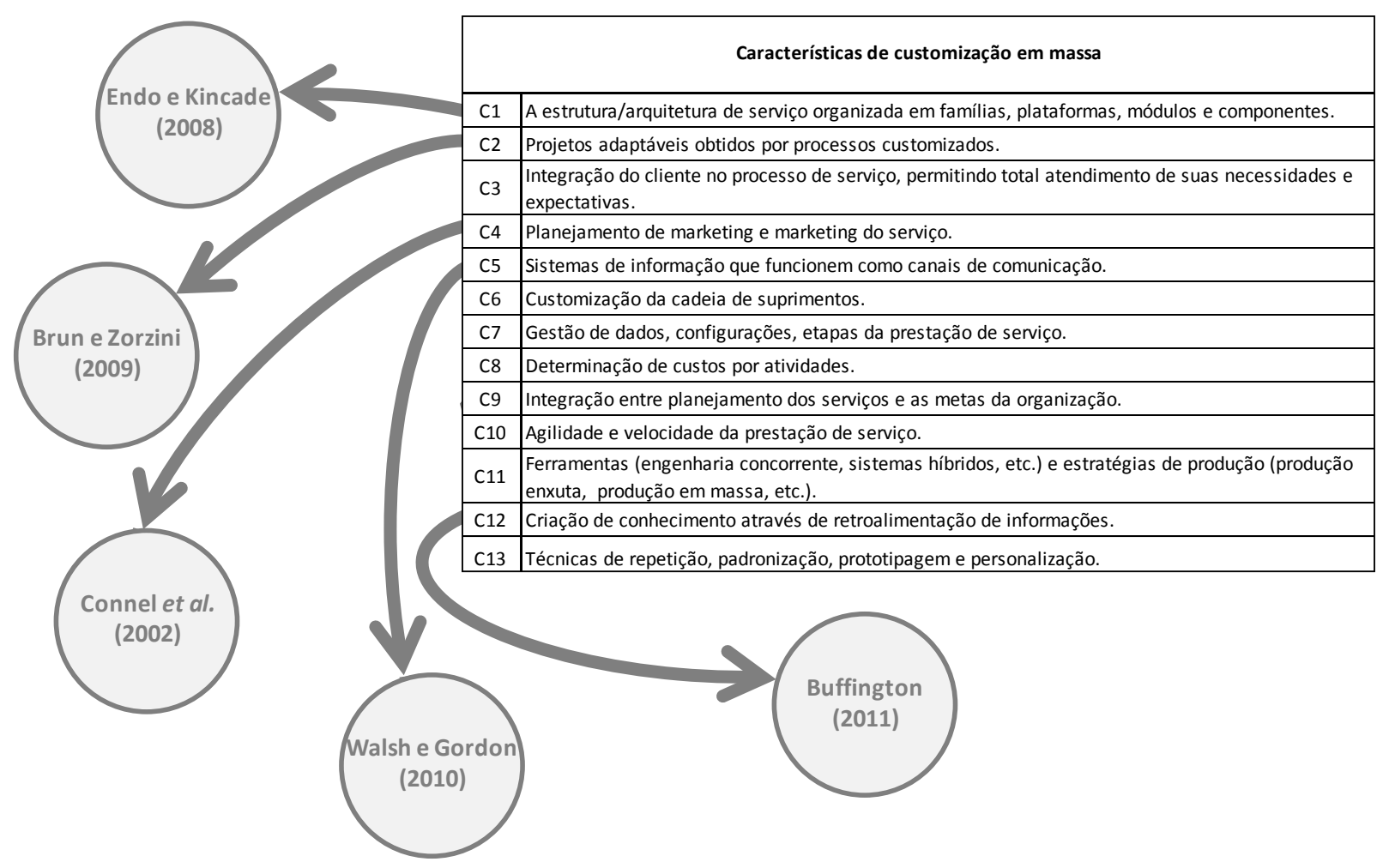

Fonte: Elaborado pelos autores

\subsection{Casos de análise e implicações gerenciais}

Os casos usados para testar o modelo são de distintas áreas de serviços. Um dos casos foi realizado com o setor de obras de uma fornecedora de energia elétrica. Esse setor caracteriza-se pelo alto volume de obras executadas mensalmente e a customização de cada uma delas. Por exemplo, dentro de um mesmo mês existem obras de construção de uma subestação para um distrito industrial, e ao mesmo tempo a instalação de um poste luz em uma área residencial. Essas obras são customizadas a partir de um grupo de dois mil componentes, previstos em legislação nacional e normas específicas para o setor.

O segundo caso foi realizado em uma Universidade, especificamente para entender a customização de uma área ensino de cursos de curta duração e 
aperfeiçoamento. Esses cursos são normalmente indicados a profissionais que finalizaram o seu bacharelado ou licenciatura e necessitam de atualização de conhecimentos em um curto espaço de tempo (menor a seis meses). Nessa Universidade, esses cursos são modelados a partir de padrões de oferta, variando evidentemente a área de conhecimento, mas focados na manutenção de um volume semestral.

Para cada um dos casos, foi solicitado que os respondentes avaliassem o potencial de cada uma das características na customização do serviço, a fim de estabelecer uma comparação do estado da prática com o framework anteriormente apresentado. As informações estão mostradas na Figura 6.

Figura 6 - importância de cada característica para empresa customizadora

\begin{tabular}{|c|l|c|c|}
\hline \multicolumn{1}{|c|}{ Características de customização em massa } & \multicolumn{2}{c|}{$\begin{array}{c}\text { Importância para empresa } \\
\text { respondente }\end{array}$} \\
\cline { 3 - 4 } & \multicolumn{1}{|c|}{ Caso 1 } & Caso 2 \\
\hline C1 & $\begin{array}{l}\text { A estrutura/arquitetura de serviço organizada em famílias, plataformas, módulos e } \\
\text { componentes. }\end{array}$ & 5.00 & 5.00 \\
\hline C2 & Projetos adaptáveis obtidos por processos customizados. & 10.00 & 7.00 \\
\hline C3 & Integração do cliente no processo de serviço, permitindo total atendimento de suas \\
necessidades e expectativas. & 10.00 & 8.00 \\
\hline C4 & Planejamento de marketing e marketing do serviço. & 3.00 & 8.00 \\
\hline C5 & Sistemas de informação que funcionem como canais de comunicação. & 10.00 & 7.00 \\
\hline C6 & Customização da cadeia de suprimentos. & 6.00 & 5.00 \\
\hline C7 & Gestão de dados, configurações, etapas da prestação de serviço. & 9.00 & 5.00 \\
\hline C8 & Determinação de custos por atividades. & 7.00 & 4.00 \\
\hline C9 & Integração entre planejamento dos serviços e as metas da organização. & 8.00 & 5.00 \\
\hline C10 & Agilidade e velocidade da prestação de serviço. & 10.00 & 5.00 \\
\hline C11 & $\begin{array}{l}\text { Ferramentas (engenharia concorrente, sistemas híbridos, etc.) e estratégias de } \\
\text { produção (produção enxuta, produção em massa, etc.). }\end{array}$ & 7.00 \\
\hline C12 & Criação de conhecimento através de retroalimentação de informações. & 8.00 & 2.00 \\
\hline C13 & Técnicas de repetição, padronização, prototipagem e personalização. & 5.00 \\
\hline
\end{tabular}

Fonte: Elaborado pelos autores

No primeiro caso as características notadamente mais importantes são C2, C3, C5 e C10 e as menos importantes são C1, C4 e C6. Contudo, para o segundo caso, as características mais importantes são C3 e C4, e as menos importantes são C11 e C8.

A comparação entre a Figura 5 e a Figura 6 mostra que existe uma grande diferença entre aquilo que pode ser implementado e aquilo que é considerado 
importante pelas organizações. A implementação em sua totalidade pode ocorrer para as características $\mathrm{C} 1, \mathrm{C} 4, \mathrm{C} 5, \mathrm{C} 7, \mathrm{C} 10, \mathrm{C} 11, \mathrm{C} 12$. Contudo, é realmente considerado como um diferencial para ambos os casos a C5 - Sistemas de informação que funcionem como canais de comunicação. Isso mostra que apesar de fácil customização, nem sempre é de interesse das organizações customizar características de serviço. A explicação para isso pode estar no custo da customização, que comparado ao benefício não parece vantajaso para a organização.

As características propostas como customizáveis em apenas alguns níveis, em geral foram destacadas como importantes pelas empresas. Isso não é um problema para o framework, visto que a customização em apenas alguns níveis não reduz a importância da customização. Apenas ratifica a necessidade de flexibilidade que o serviço deve oferecer.

Outro aspecto a ressaltar foi que as características C6 e C9, inicialmente excluídas do framework por serem consideradas não implementáveis nos serviços customizados, foram avaliadas sem grande diferenças em relação as demais. O que mostra que novos estudos devem ser realizados, a fim de viabilizar a incorporação desses elementos no modelo proposto.

\section{CONCLUSÃO}

Este trabalho teve por objetivo verificar as relações entre as estratégias de serviços e as características de customização em massa. Para tanto foi desenvolvido um framework tendo por referência as contribuições teóricas advindas dos estudos de $\mathrm{CM}$ e dos estudos que avaliam as particularidades da gestão no setor de serviços.

O modelo de classificação proposto permitiu analisar cada característica em diferentes níveis, sendo possível discutir os impactos e classificar empresas em cada um dos blocos criados. Contudo, a variável nível de customização pode restringir a aplicação da customização em certas áreas de serviços. Como se observa no estudo,

Revista Produção Online, Florianópolis, SC, v.13, n. 3, p. 974-1001, jul./set. 2013. 
há características que não transitam sobre todos os níveis de customização, o que inibe a classificação de certos tipos de empresas ou negócios no framework proposto.

Uma contribuição do estudo está na possibilidade de utilização do framework para validação dos modelos organizacionais que estão sendo usados em organizações de serviços que já utilizam a $\mathrm{CM}$, servindo como um instrumento de avaliação. Além disso, para aquelas empresas que customizam totalmente os seus serviços, podem ser criados padrões de produtos que se estendem de pequenas adptações até a customização completa.

Acredita-se que este trabalho indica linhas de pesquisa futuras para a área de customização em serviços. A primeira contempla investigar como pode ser aproveitado o conhecimento dos clientes para flexibilizar as ofertas de um determinado serviço. A segunda envolve determinar mecanismos de aquisição de conhecimento para o processo a partir do comportamento do consumidor. A terceira considera desenvolver ferramentas para racionalizar a forma como a customização de serviços pode ser realizada.

Com base no exposto, no primeiro caso, poderia ser realizado um levantamento (survey) comparando dados de consumidores que participaram da customização em um nível alto com aqueles que participaram da customização em um nível baixo. $\mathrm{Na}$ segunda, poderia ser criado um instrumento de avaliação para medir o ponto de equilíbrio em serviços entre a participação do cliente na fase anterior à prestação do serviço versus agregação de valor no produto final. Na terceira, poderia ser realizada a criação de menus de escolha e customização para serviços, utilizando esquemas similares àqueles usados na manufatura, com posteriormente validação através da comparação entre essas áreas.

Enfim, a área de serviços ainda carece de estudos relacionados à customização em massa. O framework elaborado nesse trabalho não é uma solução definitiva, mas constitui um passo para a criação de um modelo que contemple características de customização para aplicação no setor de serviços, considerando as especificidades de seus processos.

Revista Produção Online, Florianópolis, SC, v.13, n. 3, p. 974-1001, jul./set. 2013. 


\section{REFERÊNCIAS}

ABDELKAFI, N. et al. Alignment in Mass customization. In.: FOGLIATTO, F.S., DA SILVEIRA, G. Mass customization: engineering and managing global operations. Springer Velag, 2010.

ALIZON, F. et al. T.W. Framework for product family design and development.

Concurrent Engineering: Research and Applications, v.15, n.2, p.187-199, 2007.

ANZANELLO, M. J.; FOGLIATTO, F. S. Programação de tarefas baseada em curvas de aprendizado para linhas de produção customizadas. Revista Produção Online, v.11. n.3, p. 851-870, jul. 2011. Disponível em: < http://producaoonline.org.br/rpo/article/view/955/824 >.

ARAUJO FILHO, João Tomé de; GOMES, Maria de Lourdes Barreto. A customização em massa na construção civil: um estudo no subsetor de edificações. Revista Produção Online, v.10, n. 2, p. 398-423, jun. 2010. Disponível em: < http://www.producaoonline.org.br/index.php/rpo/article/viewFile/433/690 >.

APEAGYEI, P. R.; OTIENO, R. Usability of pattern customizing technology in the achievement and testing of fit for mass customisation. Journal of Fashion Marketing and Management, v. 11, n. 3, p. 349-365, 2007.

BARDAKCI, A.; WHITELOCK, J. A comparison of customers' readiness for masscustomisation: Turkish vs British customers. European Business Review, v. 17, n. 5, p. 397-410, 2005.

. How "ready" are customers for mass customization? An exploratory investigation. European Journal of Marketing, v. 38, n. 11/12, p. 1396-1416, 2004.

Mass-customisation in marketing: the consumer perspective. Journal of Consumer Marketing, v. 20, n. 5, p. 463-479, 2003.

BARE, M.; COX, J. J. Applying principles of mass customization to improve the empirical product development process. Journal of Intelligent Manufacturing, v.19, p.565-576, 2008.

BAYRAKTAR, E.; JOTHISHANKAR, M.C.; TATOGLU, E.; WU, T. Evolution of operations management: past, present and future. Management Research News, v.30, n.11, p.843-871, 2007.

BOOMS, B.H.; BITNER, M.J. Marketing strategies and organizational structures for service firms. In: DONNELLY, J.; GEORGE, W.R. Marketing of Services. Chicago: American Marketing Association, 1981, p. 47-51. 
BROWN, S; BESSANT, J. The manufacturing strategy-capabilities links in mass customisation and agile manufacturing - an exploratory study. International Journal of Operations \& Production Management, v. 23, n. 7, p. 707-730, 2003.

BRUN, A.; ZORZINI, M. Evaluation of product customization strategies through modularization and postponement. International Journal of Productions Economics, v.120, p.205-220, 2009.

BUFFINGTON, J. Comparison of mass customization and generative customization in mass markets. Industrial Management \& Data Systems, v.111, n.1, p.41-62, 2011.

CAO, J.; WANG, J.; LAW, K.; ZHANG, S. LI, M. An interactive service customization model. Information and Software Technology, v.48, p.280-296, 2006.

CHEN, Z.; WANG, L. A generic activity-dictionarybased method for product costing in mass customization. Journal of Manufacturing Technology Management, v. 18, n. 6, p. 678-700, 2007.

CHO, H.; FIORITO, S. S. Acceptance of online customization for apparel shopping. International Journal of Retail \& Distribution Management, v. 37, n. 5, p. 389-407, 2009.

CONNELL, L. J. A.; ULRICH, P. V.; BRANNON, E. L. A consumer-driven model for mass customization in the apparel market. Journal of Fashion Marketing Management, v.6, n.3, p.240-258, 2002.

CORBETT, B.; ROSEN, D. W. A configuration design based method for platform commonization for product families. Artificial Intelligence for Engineering Design, Analysis and Manufacturing, v.18, p.21-39, 2004.

DA SILVEIRA, G., BORENSTEIN, D., FOGLIATTO, F.S. Mass customization: literature review and research directions. International Journal of Production Economics. v.72, p. 1-13, 2001.

DAHMUS, J. B.; ZUGASTI, J. G. P.; OTTO, K. N. Modular product architecture. Designs Studies, v.22, n.5, p.409-424, 2001.

DAI, Z.; SCOTT, M. J. Product platform design through sensitivity analysis and cluster analysis. International Journal of Intelligent Manufacturing, v.18, p.97-113, 2007. DU, J.; JIAO, Y.; JIAO, J. Integrated BOM and routing generator for variety synchronization in assembly-to-order production. Journal of Manufacturing Technology Management, v.16, n.2, p.233-243, 2005.

DURAY, R. Mass customization origins: mass or custom manufacturing? International Journal of Operations \& Production Management. v. 22, n. 3, p. 314-328, 2002. 
DURAY, R.; WARD, P. T.; MILLIGAN, G. W.; BERRY, W. L. Approaches to mass customization: configurations and empirical validation. Journal of Operations Management, v.18, p.605-625, 2000.

ENDO, S.; KINCADE, D. H. Mass customization for long-term relationship development: Why consumers purchase mass customized products again. Qualitative Market Research: An International Journal. v.11, n.3, p.275-294, 2008.

FENG, Y. X.; ZHENG, B.; WEI, Z.; TAN, J. R. An exploratory study of the general requirement representation model for product configuration in mass customization mode. International Journal of Advanced Manufacturing Technology, v.40, p.785796, 2008.

FIORE, A. Relationships between optimum stimulation level and willingness to use mass customization options. Journal of Fashion Marketing and Management, v. 5, n. 2, p. 91-101, 2000.

FISHER, M.; RAMDAS, K.; ULRICH, K. Component sharing in the management of product variety: a study of Automotive braking systems. Management Science, v.45, n.3, p.297-315, 1999.

FITZSIMMONS, J.A.; FITZSIMMONS, M.J. Administração de Serviços. Porto Alegre: Bookman, 2010.

FIXSON, S. K. Modularity and commonality research: past developments and future opportunities. Concurrent Engineering: Research and Applications, v.15, n.2, p.85110, 2007.

FOGLIATTO, F. S.; DA SILVEIRA, G. J. C.; BORENSTAIN, D. The mass customization decade: An updated review of the literature. International Journal of Production Economics, v.138, p.14-25, 2012.

FOGLIATTO, F. S.; SILVEIRA, G. J. C. da. Mass customization: A method for market segmentation and choice menu design. International Journal of Productions Economics, v.111, p.606-622, 2008.

FRUTOS, D. J.; BORENSTEIN, D. A framework to support customer-company interaction in mass customization environments. Computers in Industry, v.54, p115135, 2004.

GERSHENSON, J. K.; PRASAD, G. J.; ZHANG, Y. Product modularity: definitions and benefits. Journal of Engineering Design, v.14, n.3, p.295-313, 2003.

GERSHENSON, J. K. Product modularity: measures and design methods. Journal of Engineering Design, v.15, n.1, p.33-51, 2004. 
HAUG, A.; LADEBY, K.; EDWARDS, K. From engineer-to-order to mass customization. Management Research News, v.32, n.7, p.633-644, 2009.

HELO, P. T.; XU, Q. L.; KYLLÖNEM, S. J.; JIAO, R. J. Integrated Vehicle Configuration System - Connecting the domains of mass customization, v.61, p.44-52, 2010.

HOEK, R. I. V. The rediscovery of postponement a literature review and directions for research. Journal of Operations Management, v.19, p.161-184, 2001.

HUANG, G. Q.; ZHANG, X. Y.; LO, V. H. Y. Integrated configuration of platform products and supply chains for mass customization: a game-theoretic approach. IEEE

Transactions on engineering management, v.54, n.1, p.156-171, 2007.

HUANG, G. Q.; ZHANG, X. Y.; LIANG, L. Towards integrated optimal configuration of platform products, manufacturing processes, and supply chains. Journal of Operations Management, v.23, p.267-290, 2005.

HUEMER, L. Unchained from the chain: Supply management from a logistics service provider perspective. Journal of Business Research, v.65, p.258-264, 2012.

ISMAIL, H.; REID, I.; POOLTON, J.; AROKIAM, I.; MOONEY, J. How Small and Medium Enterprises Effectively Participate in the Mass Customization Game. IEEE

Transactions on engineering management, v.54, n.1, P.88-97, 2007.

JIAO, J.; TSENG, M. M. A methodology of developing product family architecture for mass customization. Journal of Intelligent Manufacturing, v.10, p.3-20, 1999.

JIAO, J.; TSENG, M. M. Customizability analysis in design for mass customization. Computer Aided Design, v.36, p.745-757, 2004.

JIAO, J.; SIMPSON, T. W., SIDDIQUE, Z. Product family design and platform-based product development: a state-of-the-art review. Journal of Intelligent Manufacturing, v.18, p.5-29, 2007.

JITPAIBOON, T.; DANGOL, R.; WALTERS, J. The study of cooperative relationships and mass customization. Management Research News, v.32, n.9, p.804-815, 2009.

JOSE, A.; TOLLENAERE, M. Modular and platform methods for product family design: literature analysis. Journal of Intelligent Manufacturing, v.16, p.371-390, 2005.

KARLSSON, A. Assembly-initiated production - a strategy for mass-customization utilizing modular, hybrid automatic production systems. Assembly Automation, v.22, n.3, p.239-247, 2002. 
KINCADE, D. H.; REGAN, C.; GIBSON, F. Y. Concurrent engineering for product development in mass customization for the apparel industry. International Journal of Operations \& Production Management, v.27, n.6, p.627-649, 2007.

KOTHA, S. Mass Customization: implementing the emerging paradigm for competitive advantage. Strategic Management Journal, v.16, p.21-42, 1995.

KOTLER, P.; KELLER, K.L. Administração de marketing. São Paulo: Pearson, 2006.

KUMAR, A. From mass customization to mass personalization: a strategic transformation. International Journal of Flexibility and Manufacturing System, v.19, p.533-547, 2008.

KUMAR, A.; GATOUFI, S.; REISMAN, A. Mass customization research: trends, directions, diffusion intensity, and taxonomic frameworks. International Journal of Flexibility and Manufacturing System, v.19, p.637-665, 2008.

LEE, N. K. S., DAI, J. B. Designing and planning of material handling systems for mass customization. In.: FOGLIATTO, F.S., DA SILVEIRA, G. Mass Customization: Engineering and Managing Global Operations. Springer Velag, 2010.

LEVITT, T. A imaginação de Marketing. São Paulo: Atlas, 1991.

LI, Yi; XUE, Deyi; GU, Peihua. Design for product adaptability. Concurrent Engineering, v. 16, n. 3, p. 221-232, Sept. 2008.

LINDQUIST, A.; BERGLUND, F.; JOHANNESSON, H. Supplier integration and communication strategies in collaborative platform development. Concurrent Engineering, v.16, n.1, p.23-35, 2008.

LIOU, J. J. H.; YEN, L.; TZENG, G. H. Using decision rules to achieve mass customization of airline services. European Journal of Operations Research, v.205, p.680-686, 2010.

LOVELOCK,C; WIRTZ,J.; HEMZO, M. Marketing de Serviços: pessoas, tecnologia e estratégias. São Paulo: Pearson, 2011.

McCARTHY, I. P., PITT, L., BERTHON, P. Service customization through dramaturgy. In.: FOGLIATTO, F.S., DA SILVEIRA, G. Mass Customization: Engineering and Managing Global Operations. Springer Velag, 2010.

MA, Y.; JIAO, J.; DENG, Y. Web Service-oriented electronic catalogs for product customization. Concurrent Engineering, v.16, n.4, p.263-270, 2008. 
MARION, T. J.; FREYER, M.; SIMPSON, T. W.; WYSK, R. A. Design for mass customization in the early stages of product development. International Design Engineering Technical Conferences \& Computers and Information in Engineering Conference, p.10-13, 2006.

MUFFATTO, M. Introducing a platform strategy in product development. International Journal of Production Economics, v.60-61, p.145-153, 1999.

MUN, D.; JANG, K.; HAN, S.; KIM, J.; HWANG, H. Engineered-to-order approach for providing flexibility in e-commerce of mold parts. Concurrent Engineering, v.15, n.4, p.345-355, 2007.

NI, Q. F.; LU, W. F.; YARLAGADDA, P. K. D. V. An Extensible product structure model for product lifecycle management in the make-to-order environment. Concurrent Engineering, v.16, n.4, p.243-251, 2008.

PAN, B., HOLLAND, R. A mass customized supply chain for the fashion system at the design production interface. Journal of Fashion Marketing and Management, v.10, n.3, p.345-359, 2006.

PETERS, L.; SAIDIN, H. IT and the mass customization of services: the challenge of implementation. International Journal of Information Management, v.20, p.103-119, 2000.

PRIETO, E.; LAURINDO,F. J. B.; CARVALHO, M. M. Alinhamento entre a estratégia do negócio e a gestão do relacionamento com o cliente: caso de uma empresa de telefonia. Revista Produção Online, v.7, n.2, p. 30-42, ago. 2007. Disponível em: $<$ http://producaoonline.org.br/rpo/article/view/76/80>.

PILLER, F. T.; MOESLEIN, K.; STOTKO, C. M. Does mass customization pay? An economic approach to evaluate customer integration. Production Planning and Control, v.15, n.4, p.435-444, 2004.

PILLER, F.T. Mass Customization: Reflections on the state of the concept. International Journal of Flexible Manufacturing Systems, v.16, p.313-334, 2004.

PILLER, F. T. Observations on the present and future of mass customization. International Journal of Flexibility and Manufacturing System, v.19, p.630-636, 2008.

REUVER, M.; BOUWMAN, H. Governance mechanisms for mobile service innovation in value networks. Journal of Business Research, v. 65, p. 347-354, 2012. 
SALVADOR, F.; MARTIN, P.; PILLER, F. Decodificando a customização em massa. HSM Management, v.6, n.77, p.140-148, 2009.

SALVADOR, F.; FORZA, C.; RUNGTUSANATHAM, M. Modularity, product variety, production volume, and component sourcing: theorizing beyond generic prescriptions. Journal of Operations Management, v.20, p.549-575, 2002.

SIDDIQUE, Z.; BODDU, K. R. A mass customization information framework for integration of customer in the configuration/design of a customized product. Artificial Intelligence for Engineering Design, Analysis and Manufacturing, v.18, p.71-85, 2004.

SIGALA, M. Mass customization implementation models and customer value in mobile phones services: preliminary findings from Greece. Managing Service Quality, v.16, n.4, p.395-420, 2006.

SIMPSON, T. W.; MARION, T.; WECK, O.; OTTO, K. H.; KOKKOLARAS, M.; SHOOTER, S. B. Platform-based design and development: current trends and needs in industry. International Design Engineering Technical Conferences \& Computers and Information in Engineering Conference, 2006.

SIMPSON, T.W.; MAIER, J. R. A.; MISTREE, F. Product platform design: method and application. Research Engineering Design, v.13, p.2-22, 2001.

SIMPSON, T. W. Product platform design and customization: status and promise. Artificial Intelligence for Engineering Design, Analysis and Manufacturing, v.18, p.3-20, 2004.

SPRING, M., ARAUJO, L. Service, services and products: rethinking operations strategy. International Journal of Operations \& Production Management. v. 29, n. 5, p. 444-467, 2009.

STARR, M. K. Modular production - a 45-year-old concept. International Journal of Operations \& Production Management, v.30, n.1, p. 7-19, 2010.

THEVENOT, H. J.; SIMPSON, T. W. Commonality indices for product family design: a detailed comparison. Journal of Engineering Design, v.17, n.2, p.99-119, 2006.

TRENTIN. A.; PERIN, E.; FORZA, C. Product configurator impact on product quality. International Journal of Production Economics, v.135, p.850-859, 2012.

TSENG, M. M.; JIAO, J. Design for Mass Customization. Annals of the CIRP, v.45, n.1, p.153-156, 1996. 
TSENG, M. M., RADKE, A. M. Production planning and control for mass customization. In.: FOGLIATTO, F.S., DA SILVEIRA, G. Mass Customization: Engineering and Managing Global Operations. Springer Velag, 2010.

WALSH, K.; GORDON, J. R. Understanding professional service delivery. International Journal of Quality and Service Sciences, v.2, n.2, p.217-238, 2010.

WANG, H.; LIN, Z. Defects tracking matrix for mass customization production based on house of quality. International Journal of Flexibility and Manufacturing System, v.19, p.666-684, 2008.

WILLIAMS, C. B.; ALLEN, J. K.; ROSEN, D. W.; MISTREE, F. Designing platforms for customizable products and processes in markets of non-uniform demand. Concurrent Engineering, v.15, n.2, p.201-216, 2007.

YANG, B.; BURNS, N. D.; BACKHOUSE, C. J. Postponement: a review and an integrated framework. International Journal of Operations \& Production Management, v.24, n.5, p.468-487, 2004.

ZHA, X. F.; SRIRAM, R. D.; LU, W. F. Evaluation and selection in product design for mass customization: a knowledge decision support approach. Artificial Intelligence for Engineering Design, Analysis and Manufacturing, v.18, p.87-109, 2004.

ZAHAJ, D; GRIFFIN, A. Are customer information systems worth it? Results from B2B services. Marketing Science Institute Working Paper, 2002.

ZEITHAML, V.A. How consumer evaluation process differ betwenn goods and services. In: DONNELLY, J.; GEORGE, W.R. Marketing of Services. Chicago: American Marketing Association, 1981, p. 186-190.

ZHANG, X., CHEN, R. Forecast-driven or customer-order-driven? An empirical analysis of the Chinese automotive industry. International Journal of Operations \& Production Management. v. 26, n. 6, p. 668-688, 2006.

ZHANG, W. Y.; TOR, S, Y.; BRITTON, G. A. Managing modularity in product family design with functional modeling. International Journal of Advanced Manufacturing Technology, v.30, p.579-588, 2005.

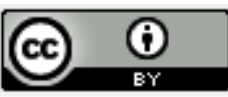

Artigo recebido em 09/06/2012 e aceito para publicação em 01/11/2012.

Revista Produção Online, Florianópolis, SC, v.13, n. 3, p. 974-1001, jul./set. 2013. 